\title{
Dual-task cost in people with multiple sclerosis: A case-control study
}

British Journal of Occupational Therapy 2018, Vol. 81(7) 384-392

(C) The Author(s) 2018

Reprints and permissions:

sagepub.co.uk/journalsPermissions.nav

DOI: $10.1177 / 0308022618757936$

journals.sagepub.com/home/bjot

\author{
Jolien Lemmens ${ }^{1}$, Sofie Ferdinand ${ }^{2}$, Annick Vandenbroucke ${ }^{3}$, \\ Stephan Ilsbroukx ${ }^{4}$ and Daphne Kos ${ }^{5}$
}

\begin{abstract}
Introduction: Limitations in fine motor functioning and cognitive performance are frequently reported in multiple sclerosis. Studies about dual-task costs in multiple sclerosis generally explore walking and postural performance. This study aims at exploring dual-task costs when simultaneously performing fine motor and cognitive tasks.

Method: Eighty people with multiple sclerosis and 80 matched controls performed the nine-hole peg test and the months backward test under single and dual-task conditions. Differences in performance over time were analysed with the paired $t$-test; differences in dual-task costs between groups were analysed with independent $t$-tests.

Results: People with multiple sclerosis and controls showed a significant decrease in fine motor and cognitive performance in dual-task conditions compared to single-task conditions $(P<0.01)$. People with multiple sclerosis with limited hand capacity showed larger dual-task costs for cognitive performance compared to other hand capacity groups $(P<0.05)$. Individuals with multiple sclerosis with lower cognitive capacity showed larger dual-task costs for fine motor performance $(P<0.01)$.

Conclusion: Both people with multiple sclerosis and controls experience dual-task costs. People with multiple sclerosis with limited hand function showed an increased dual-task cost of cognitive performance and individuals with limited cognitive capacity showed a higher dual-task cost for fine motor performance. Therefore, occupational therapists should focus on cognitive or fine motor aspects during dual-task training dependent on individuals' capacities.
\end{abstract}

\section{Keywords}

Dual-task cost, task performance, multiple sclerosis, nine-hole peg test, months backwards test

Received: 7 February 2017; accepted: 15 January 2018

\section{Introduction}

Multiple sclerosis (MS) is a progressive disease of the central nervous system that damages the myelin as well as the nerve fibres themselves. This damage leads to a variety of symptoms, such as deficits in gait, balance and cognitive performance (Bjartmar and Trapp, 2001). Of the individuals with MS who experienced walking difficulties, about $70 \%$ reported gait dysfunction as a major challenge (LaRocca, 2011). Cognitive dysfunction appears in $40-70 \%$ of people with MS and can be reflected in information processing speed, memory, verbal fluency and executive functions (DeLuca et al., 2015). In addition, people with MS may have reduced upper limb and fine motor performance, affecting daily life functioning (Bertoni et al., 2015; Lamers et al., 2013).

It is hypothesized that people with MS have an increased dual-task cost (DTC) in simultaneously performing two tasks, also known as a dual task. The DTC is the decline of a performance score of a certain task if that activity is simultaneously performed with another task. The DTC may hinder daily life activities, such as walking while talking (on the phone) with a friend, with a potential risk of falling (Wajda et al., 2013). In healthy people DTCs of motor and cognitive performance increase with age, which is potentially related to the sensory and motor aspects of behavior and adaptive strategies needed for cognitive control (Lindenberger et al., 2000; Malcolm et al., 2015). The neural deficits related to MS may lead to an increased brain area activation to perform motor and cognitive tasks in single-task performance, which is even more obvious in dual-task circumstances compared to healthy controls (Downer et al., 2016).

${ }^{1}$ Occupational Therapist, Sint-Gerardus, Diepenbeek and University College PXL, Hasselt, Belgium; previous research student, KU Leuven (Rehabilitation Sciences), Leuven and University of Ghent (Faculty of Medicine and Health Sciences), Ghent, Belgium

${ }^{2}$ Head of Occupational Therapy Department, National Multiple Sclerosis Center, Melsbroek, Belgium

${ }^{3}$ Occupational Therapist, National Multiple Sclerosis Center, Melsbroek, Belgium

${ }^{4}$ Rehabilitation Physician, Hospital Gasthuis Zusters Antwerpen (GZA), Antwerp, Belgium

${ }^{5}$ Professor in Occupational Therapy, KU Leuven, Leuven and AP University College Antwerp, Belgium

Corresponding author:

Daphne Kos, Department of Rehabilitation Sciences, Tervuursevest 101 box 1501, 3001 Leuven, Belgium.

Email: daphne.kos@kuleuven.be 
Theoretical models may explain the occurrence of DTsC (Tombu and Jolicœur, 2003). The bottleneck model assumes mental operations that must be carried out sequentially, that is if the operation requires the performance of two tasks at a single point in time, the different neural paths might inhibit each other (Pashler, 1994). The capacity-sharing model is based on the idea that the brain is running on mental fuel that can be distributed among different tasks (Tombu and Jolicœur, 2003). When this fuel is devoted to one certain task, there is less for others. The third model, the cross-talk model, suggests that when the dual task contains two completely different tasks, the interference is lower compared with two similar tasks (Navon and Miller, 1987).

Although the DTC of walking and postural performance in people with MS is widely explored, less is known about the DTC of cognitive and fine motor performance (Wajda and Sosnoff, 2015). In the systematic review of Wajda and Sosnoff (2015), 20 papers focusing on the DTC during walking and balance tasks in people with MS were included. The review revealed evidence for motor interference of walking speed and balance in dual-task conditions in individuals with MS, but limited evidence for the effect of dual tasking on cognitive and fine motor performance. Only one study investigated the DTC of fine motor performance and found significant differences in the DTC for people with different disability levels (Learmonth et al., 2015). Studies exploring the DTCs among different disability levels mainly use the Expanded Disability Status Scale (EDSS), a method of quantifying the gross motor disability in people with MS. Studies investigating the DTC of fine motor and cognitive performance should base their research on other criteria. For example, comparing groups based on hand or cognitive capacity can expand our knowledge about the DTCs among different domains of disability. This, in turn, can clarify the need and possibilities of dual-task training, because even the most severely damaged patients are capable of performance improvements (Tomassini et al., 2011).

Because the DTC may be an indicator for difficulties in performing daily life activities requiring fine motor function that are not visible in single-task situations, it is warranted to explore the DTC in fine motor and cognitive performance in MS and healthy controls. The results of this study may support the development of dual-task training among persons with MS. Therefore, this study will answer the following primary research question:

Is a DTC of fine motor and cognitive performance present in people with MS and does this differ from healthy controls?

To support the developmental process of dual-task training, it is indicated to investigate which task is prioritized. Therefore, the following secondary research questions will also be answered in this study:

Which task do the participants prioritize within the dual task? Is there a difference in prioritization between the MS group and healthy controls? Which variables play a role in the DTC of fine motor and/or cognitive performance?

\section{Method}

This article is reported according to the STROBE checklist (von Elm et al., 2008).

\section{Sample}

This case-control study was approved by the local ethical committee of the National MS Centre Melsbroek, Belgium (2016) and the ethical committee of the University Hospital Ghent, Belgium (2016, reference 2015/1503). All participants provided written informed consent.

This study was conducted in the National MS Centre Melsbroek from September 2015 to March 2016. A convenience sample of 80 individuals with MS visiting the MS rehabilitation center (inpatients and outpatients) and 80 healthy controls was selected. Sample size calculation was based on the DTCs found in a pilot study, using statistical power analysis software $\mathrm{G}^{*}$ power 3.1 (requiring $80 \%$ power and providing the effect size of 0.61 of the difference in the DTC of the nine-hole peg test (NHPT) between MS and controls), indicating a minimum of $35 \mathrm{sub}-$ jects per group (Faul et al., 2007; Ferdinand et al., 2015). The investigator recruited the participants and administered the tests in the MS center. A convenience sample of healthy age and gender matched controls was found among family members and neighbors of the investigator. For every person with MS, a control person of the same gender and the same age group was found. Every assessed MS participant was allocated to an age group. Every age group contained 5 years, resulting in 11 age groups, both for men and women, ranging from 21 to 75 years.

Inclusion criteria were chosen in line with previous research investigating DTCs in MS and the pilot study (Ferdinand et al., 2015; Wajda and Sosnoff, 2015). The participants with MS were included when the following criteria were met: definite diagnosis of MS (Hawkes and Giovannoni, 2010; Polman et al., 2005); NHPT (Mathiowetz et al., 1985) feasible within two minutes; brief repeatable battery for MS (BRB) (Rao, 1990) score of at least 3 out of 4 ; months backward test (MBT) (Cunningham et al., 2015) performed with less than three errors; relapse-free for 30 days prior to participation; native language was Dutch, French, English or German; understanding verbal instructions in the Dutch language.

To determine whether the participants met the inclusion criteria, the investigator checked electronic patient files about the diagnosis, NHPT score, BRB Rao score, relapses and native language. Rao's BRB is a method to assess the cognition of patients with MS in the daily clinic. It assesses attention, visual precision, executive functions, verbal memory, delayed recall, visual-spatial memory, semantic retrieval and verbal fluency. A score of 3 or 4 means there are no severe cognitive deficits (Boringa et al., 2001). An understanding of the Dutch language was based on a first conversation and impression.

Because this study aims to investigate the DTC among MS and healthy controls, participants with other diseases such as a rheumatic disease or a disease of the central/ 
peripheral nervous system (other than MS) were excluded from this study. Also, participants with an extremely lowered vision were excluded because they must be capable to perform the NHPT.

\section{Data collection}

Practice sessions. The participants first performed the MBT three times to minimize learning effects afterwards (Cunningham et al., 2015). The MBT is a measure of attention in which the participants recite the months in reverse order and evaluates different aspects of cognition, namely attention, working memory, executive function and cognitive flexibility. The MBT has good concurrent validity and excellent test-retest reliability (Östberg et al., 2012). In these practice sessions, the duration of reciting the months three times consecutively was recorded.

Testing. The order of the three test conditions was randomized by way of a $3 \times 3$ Latin square for each participant including all combinations of the NHPT (A), the MBT (B) and the dual-task conditions (C).

The NHPT is a fine motor test in which the person must place nine pegs one at a time in the commercially available square board with nine holes (Mathiowetz et al., 1985). Then the person must remove the pegs and return them to the container. Timing should be performed with a stopwatch and recorded in seconds. This procedure is repeated twice for both hands. The NHPT was performed according to the standardized instructions. The NHPT has excellent intra and interrater reliability, good criterion validity, adequate floor effects and a high responsiveness in people with MS (Lamers et al., 2014). Hand dominance was based on self-report.

The participants performed the MBT by reciting the months in reverse order as many times as possible during 30 seconds, as fast and correctly as possible. The MBT accuracy and the MBT speed were measured.

Participants performed the dual task in which the NHPT was conducted simultaneously with the MBT. The MBT continued until the end of the NHPT. The participants received the instruction to recite as many months as possible during the NHPT. The participant was allowed to choose to name a month and put a peg into the board at the same time or at an alternating rhythm.

\section{Statistical analysis}

The analysis was performed using SPSS 22 software (SPSS Inc., Chicago, IL, USA). To calculate the DTC of fine motor and/or cognitive performance, the following calculation was used: $\mathrm{DTC}=($ single task - dual task $) /$ single task $\times 100$ (Leone et al., 2015).

Six different DTCs were calculated for every participant (Table 1). For statistical analysis, the mean of every DTC was calculated within each group (MS versus healthy controls). To explore differences among hand capacity groups, the third quartile of the NHPT scores in singletask conditions was used. Because upper limb function can
Table 1. Explanation of different dual-task costs (DTCs).

\begin{tabular}{lc}
\hline DTC & Explanation \\
\hline DTC NHPT D & $\begin{array}{c}\text { Decline in fine motor performance of } \\
\text { dominant hand in a dual-task condition } \\
\text { compared to a single-task condition } \\
\text { Decline in fine motor performance of } \\
\text { non-dominant hand in a dual-task } \\
\text { condition compared to a single-task } \\
\text { condition }\end{array}$ \\
DTC NHPT ND & $\begin{array}{c}\text { Reduction of MBT speed in a dual-task } \\
\text { condition (during NHPT with dominant } \\
\text { hand) compared to a single-task } \\
\text { condition }\end{array}$ \\
DTC MBT speed & $\begin{array}{c}\text { Reduction of MBT accuracy in a dual-task } \\
\text { condition (during NHPT with dominant } \\
\text { during NHPT D } \\
\text { hand) compared to a single-task } \\
\text { condition }\end{array}$ \\
DTC MBT accuracy & $\begin{array}{c}\text { Reduction of MBT speed in a dual-task } \\
\text { condition (during NHPT with } \\
\text { during NHPT D } \\
\text { non-dominant hand) compared to a } \\
\text { single-task condition }\end{array}$ \\
DTC MBT speed & $\begin{array}{c}\text { Reduction of MBT accuracy in a dual-task } \\
\text { condition (during NHPT with } \\
\text { during NHPT ND } \\
\text { non-dominant hand) compared to a } \\
\text { single-task condition }\end{array}$ \\
\hline
\end{tabular}

NHPT: nine-hole peg test; MBT: months backward test; D: dominant hand, ND: non-dominant hand.

be affected asymmetrically in MS (Heremans et al., 2012), two cut-off scores were determined: one for the dominant (33.5 seconds) and one for the non-dominant hand (37.2 seconds). Participants with a NHPT score equal or higher than cut-off, were categorized in the lower hand capacity group. In controls, the cut-off scores were 20 seconds for the dominant hand and 21.8 seconds for the non-dominant hand. For cognitive capacity groups, the first quartiles of the baseline MBT speed scores (months per second) ( 0.70 for MS and 0.88 for controls) were used as the cut-off.

Independent $t$-tests or chi-square tests were used to investigate differences in demographics and scores between MS and controls and between capacity groups, based on the measurement level of the variable. Paired $t$-tests were used to examine the DTC for fine motor and cognitive performance and to explore the learning effect of the MBT. A repeated measures analysis of variance was performed to explore differences in learning effects of the MBT between MS and the control group. The chisquare test was used to investigate prioritization.

A stepwise multiple linear regression analysis was conducted for all participants and for MS and control groups separately to investigate which variables are associated with DTCs. The independent variables were the presence/absence of MS diagnosis, age, gender, dominance and the three baseline scores of the MBT. Variables were included when $F$ increased by at least 0.05 and were excluded if $F$ increased by less than 0.1 . The dependent variables were the six DTCs. Multicollinearity was present when the tolerance value was lower than 0.1 . The alpha level was set at 0.05 . Missing data were not analysed. 


\section{Results}

The sample included 80 participants with MS and 80 healthy controls with a mean age of 53 years (SD 9.4) and a $65 \%$ female/male ratio (Table 2). Mean scores of the NHPT and the MBT were significantly lower in the MS group. Three baseline MBT scores significantly improved over time $(P<0.001)$ in both groups (time*group interaction $P=0.71$ ), suggesting an overall learning effect. Single-task performance of the MBT (speed) and the NHPT of controls is in line with norms for healthy adults with similar age profiles; the MS sample performed both tests at a slower speed (Mathiowetz et al., 1985; Östberg et al., 2012).

Individuals showed a significantly lower fine motor performance (mean NHPT score) and cognitive performance (MBT speed) in dual-task conditions compared to single-task conditions, indicating a DTC in both variables (Table 2 (A)). In contrast, accuracy scores of the MBT in single and dual-task conditions did not significantly differ ( $P=0.32$ in the dominant hand; $P=0.94$ for the non-dominant side). The DTCs of MBT accuracy scores were therefore not included for further analyses.

The means of the different DTCs were not significantly different between MS participants and controls, nor between hand dominance groups (Table 2 (B)).

In the MS group, significant differences between lower and higher hand capacity groups were found in the DTC of MBT speed ( $P=0.01$ for the dominant hand; $P=0.02$ for the non-dominant hand) (Table 3 ). In addition, a significant difference has been found in the DTC of NHPT speed between non-dominant hand capacity groups $(P<0.01)$. In the latter case, the results were in advantage for the lower hand capacity group.

The results indicate that individuals with MS with highly affected hand capacity performed worse on the MBT when this task was executed simultaneously with a fine motor task. Among control participants, for none of

Table 2. Demographic and clinical data of baseline and dual-task assessment.

\begin{tabular}{|c|c|c|c|}
\hline Variable & MS $(n=80)$ & Controls $(n=80)$ & $\begin{array}{l}\text { Difference between } \\
\text { groups }(P)\end{array}$ \\
\hline \multicolumn{4}{|l|}{ Baseline data } \\
\hline Age (years; mean (SD)) & $53.43(9.41)$ & $53.24(9.44)$ & 0.9 \\
\hline Gender (women; \%, $n$ ) & $65 ; 52$ & $65 ; 52$ & 1 \\
\hline Dominance (dextral; \%, $n$ ) & $93.8 ; 75$ & $93.8 ; 75$ & 1 \\
\hline Baseline NHPT D (s; mean (SD)) & $30.63(16.88)$ & $18.43(2.51)$ & $<0.001$ \\
\hline Baseline NHPT ND (s; mean (SD)) & $32.15(16.92)$ & $19.72(2.69)$ & $<0.001$ \\
\hline Baseline 1 MBT (s; mean (SD)) & $49.18(19.8)$ & $39.19(12.91)$ & $<0.001$ \\
\hline Baseline 2 MBT (s; mean (SD)) & $45.1(16.74)$ & $36.41(11.35)$ & $<0.001$ \\
\hline Baseline 3 MBT (s; mean (SD)) & $43.81(16.2)$ & $35.02(10.07)$ & $<0.001$ \\
\hline \multicolumn{4}{|l|}{ Dual-task results } \\
\hline $\begin{array}{l}\text { A. Mean Difference Between Single And Dual Task Scores } \\
\text { (mean }(95 \% \mathrm{Cl}))\end{array}$ & MS & Controls & \\
\hline NHPT D (s) & $-6.9(-9.0 ;-4.8)$ & $-5.8(-6.9 ;-4.7)$ & \\
\hline NHPT ND (s) & $-6.3(-8.2 ;-4.4)$ & $-4.7(-5.8 ;-3.7)$ & \\
\hline MBT speed D (months/s) & $0.4(0.3 ; 0.4)$ & $0.4(0.4 ; 0.4)$ & \\
\hline MBT speed ND (months/s) & $0.3(0.3 ; 0.4)$ & $0.3(0.3 ; 0.4)$ & \\
\hline MBT accuracy D (number of errors) & $0.1(-0.1 ; 0.2)$ & $0.1(-0.1 ; 0.2)$ & \\
\hline MBT accuracy ND (number of errors) & $-0.0(-0.2 ; 0.1)$ & $0.0(-0.2 ; 0.2)$ & \\
\hline B. Dual-task cost (DTC) (\%) & MS & Controls & $\begin{array}{l}\text { Mean difference between } \\
\text { MS and controls }(95 \% \mathrm{Cl})\end{array}$ \\
\hline DTC NHPT D (s) & -25.69 & -31.87 & $6.2(-2.9 ; 15.2)$ \\
\hline DTC NHPT ND (s) & -22.68 & -23.88 & $1.2(-6.4 ; 8.8)$ \\
\hline DTC MBT speed D (months/s) & 35.92 & 31.88 & $4.0(-1.2 ; 9.3)$ \\
\hline \multirow[t]{2}{*}{ DTC MBT speed ND (months/s) } & 32.91 & 27.79 & $5.1(-0.4 ; 10.5)$ \\
\hline & $\begin{array}{l}\text { Right dominant } \\
(n=150)\end{array}$ & $\begin{array}{l}\text { Left dominant } \\
(n=10)\end{array}$ & $\begin{array}{l}\text { Mean difference between } \\
\text { dexterity groups }(95 \% \mathrm{Cl})\end{array}$ \\
\hline DTC NHPT D (s) & -28.8 & -29.6 & $0.8(-17.9 ; 19.6)$ \\
\hline DTC NHPT ND (s) & -23.2 & 23.9 & $0.7(-14.9 ; 16.3)$ \\
\hline DTC MBT speed D (months/s) & 33.7 & 36.1 & $-2.4(-13.2 ; 8.5)$ \\
\hline DTC MBT speed ND (months/s) & 30.1 & 33.8 & $-3.7(-15.1 ; 7.7)$ \\
\hline
\end{tabular}

MS: multiple sclerosis; NHPT D: nine-hole peg test performed with dominant hand; NHPT ND: nine-hole peg test performed with non-dominant hand; Baseline 1, 2 and 3: practice sessions of MBT; MBT: months backward test; DTC: dual-task cost (calculation: see text); $95 \%$ Cl: $95 \%$ confidence interval. 
Table 3. Comparison of different dual-task costs (DTCS) (mean (SD)) between hand capacity groups among MS participants and healthy controls.

\begin{tabular}{lllr}
\hline DTC & DTC Mean (SD) & & $P$ value \\
\hline MS participants & & & \\
& Lower hand capacity group $(n=20)$ & Higher hand capacity group $(n=59)$ & \\
DTC NHPT & D: $-20.19(27.42)$ & D: $-27.56(30.28)$ & 0.47 \\
& ND: $-12.02(23.22)$ & ND: $-26.25(24.62)$ & 0.01 \\
DTC MBT & D: $43.31(17.59)$ & D: $32.96(14.04)$ & 0.02 \\
& ND: $40.34(17.02)$ & ND: $30.46(17.25)$ & \\
Control participants & & & \\
\multirow{2}{*}{ DTC NHPT } & Lower hand capacity group $(n=20)$ & Higher hand capacity group $(n=60)$ & 0.07 \\
& D: $-23.73(19.18)$ & D: $-34.59(30.22)$ & 0.89 \\
DTC MBT & ND: $-23.26(23.98)$ & ND: $-24.08(23.53)$ & 0.97 \\
& D: $31(14.55)$ & D: $31.82(15.47)$ & 0.91 \\
\hline
\end{tabular}

MS: multiple sclerosis; D: dominant hand; ND: non-dominant hand; DTC NHPT: dual-task cost for fine motor performance; DTC MBT: dual-task cost for cognitive performance.

the DTCs were significant differences found between hand capacity groups.

\section{Comparison of DTCs between cognitive capacity groups}

Individuals with MS with highly affected cognitive capacity performed worse on the NHPT in dual-task conditions compared to individuals with a higher cognitive capacity in both hands $(P<0.01)$ (Figure $1(\mathrm{a}))$. Among controls, the DTCs for fine motor performance were not significantly different from each other (Figure 1(b)).

\section{Investigation of prioritizing}

To investigate which task was prioritized, the number of participants who had a dual-task win (DTW) on the NHPT or the MBT were selected. A DTW is a gain of performance in dual-task conditions compared to the single-task conditions, i.e. when the participant has a higher performance in dual-task conditions compared to the single-task conditions, which is not common. Results showed no difference in DTW frequencies between MS and controls, indicating that prioritizing was not depending on the absence or presence of MS diagnosis. There was a significant difference between DTW frequencies in advantage of the NHPT conducted with the dominant hand $(P<0.01)$. When the fine motor task was conducted by the non-dominant hand, there was no difference in prioritizing $(P=0.12)$.

\section{Associating variables of the DTC}

The results of all regression analyses are displayed in Table 4. No issues with collinearity were present in any of the variables (all tolerance values were above 0.1) (results not shown).

Among all participants, the multiple regression analyses revealed that within different DTCs, the presence of
MS (DTC of the NHPT dominant hand) and first MBT baseline (DTC of the NHPT non-dominant hand and DTC of the MBT non-dominant hand) were significant coefficients. Within the separate models for the MS and control group, one or more of the three baseline scores of the mental task explained the variability in DTCs by $6-25 \%$.

\section{Discussion and implications}

This study investigated the DTC of fine motor and cognitive performance among individuals with MS and between hand capacity and cognitive capacity groups. It also studied the prioritizing of dual tasking and variables that are associated with DTCs.

When the NHPT and the MBT were simultaneously performed, almost all participants showed a decreased fine motor (NHPT speed) and/or reduced MBT speed in a dual-task condition compared to a single-task condition. Participants did not make significantly more errors during the MBT in a dual-task condition compared to a singletask condition, indicating that MBT accuracy was not affected by a dual-task condition. Although single-task performance differed between individuals with MS and controls, the DTCs were not different. This is in line with previous research using mental tracking tasks combined with tasks of walking or postural control, although it is unclear whether performances in both groups are comparable at the neural activation level (Learmonth et al., 2017). When specifically looking at individuals with MS in the lower hand capacity group, a more pronounced dual-task effect was visible in MBT speed when simultaneously performed with the NHPT. These differences were not found among healthy controls, suggesting brain damage and related disability level impacts performance in a dual-task condition. Only one study also found significant differences in the DTC for people with different disability levels based on the EDSS (Learmonth et al., 2015). Other research showed no 

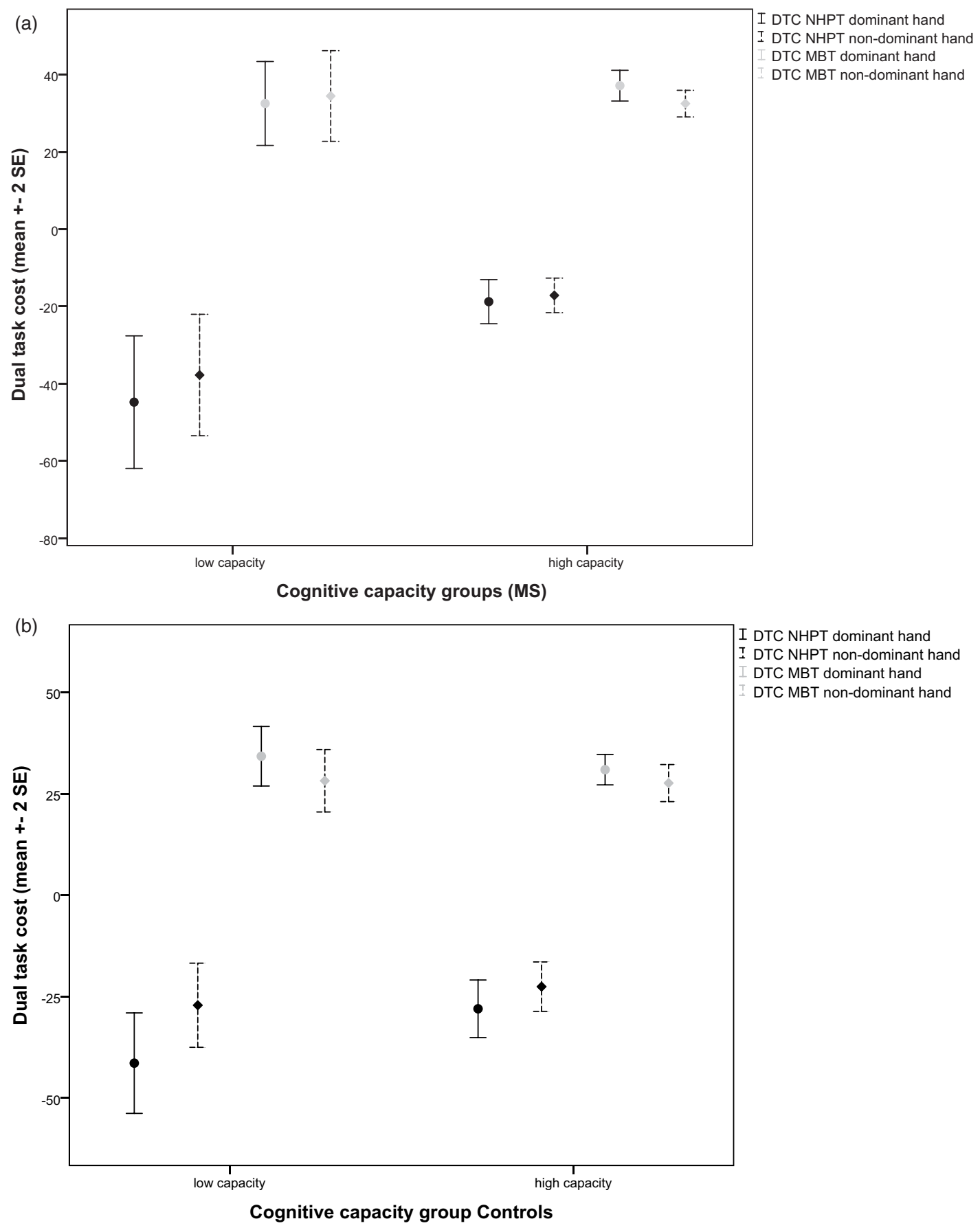

Figure 1. Dual-task cost (DTC) (\%) of fine motor (nine-hole peg test (NHPT), seconds) and cognitive performance (months backward test (MBT), months/second) among cognitive capacity groups in the multiple sclerosis (MS) group (a) and healthy controls (b). (a) MS group. Note the significant difference between the DTC for fine motor performance (NHPT dominant and non-dominant hand) among cognitive capacity groups in MS. (b) Healthy controls. Note: there are no significant effects between any DTC for cognitive capacity groups among healthy controls.

distinction between disability level groups based on the EDSS, although most studies included people with mild disability (Learmonth et al., 2017). Yet no other studies have made a distinction in hand capacity levels.

Participants with MS who performed worse on the MBT task in a single-task condition (lower cognitive capacity group), showed a larger DTC for fine motor performance in both hands. To our knowledge, no other studies have reported on DTC differences among various baseline cognitive levels. However, this is an important finding as it suggests that reduced cognitive capacity may also impact fine motor performance in real-life situations in which dual tasking is more common than labbased testing without distraction.

MS individuals and healthy controls did not differ in prioritizing a certain task. Participants prioritized the fine motor task above the mental task when performed by the dominant hand. The prioritization is influenced by the 
Table 4. Results of regression analyses.

\begin{tabular}{|c|c|c|c|c|c|}
\hline Dependent variable & Coefficients & $R^{2}$ & B & $\begin{array}{l}\text { Standard } \\
\text { error }\end{array}$ & $P$ value \\
\hline \multicolumn{6}{|l|}{ Total sample } \\
\hline \multirow[t]{2}{*}{ DTC NHPT dominant hand } & & 0.10 & & & \\
\hline & Presence of MS & & -11.24 & 4.59 & 0.02 \\
\hline \multirow[t]{2}{*}{ DTC NHPT non-dominant hand } & & 0.11 & & & \\
\hline & MBT_1 & & -0.46 & 0.1 & $<0.001$ \\
\hline DTC MBT dominant hand & & $\begin{array}{l}\text { No variables entered } \\
\text { in the model }\end{array}$ & & & \\
\hline \multirow[t]{2}{*}{ DTC MBT non-dominant hand } & & 0.05 & & & \\
\hline & MBT_1 & & 0.23 & 0.08 & 0.003 \\
\hline \multicolumn{6}{|l|}{ Participants with MS } \\
\hline \multirow[t]{2}{*}{ DTC NHPT dominant hand } & & 0.07 & & & \\
\hline & MBT_2 & & -0.46 & 0.19 & 0.02 \\
\hline \multirow[t]{3}{*}{ DTC NHPT non-dominant hand } & & 0.25 & & & \\
\hline & MBT_3 & & -1.28 & 0.31 & $<0.001$ \\
\hline & MBT_2 & & 0.64 & 0.30 & 0.04 \\
\hline DTC MBT dominant hand & & $\begin{array}{l}\text { No variables entered } \\
\text { in the model }\end{array}$ & & & \\
\hline \multirow[t]{2}{*}{ DTC MBT non-dominant hand } & & 0.06 & & & \\
\hline & MBT_1 & & 0.21 & 0.10 & 0.03 \\
\hline \multicolumn{6}{|l|}{ Healthy controls } \\
\hline \multirow[t]{2}{*}{ DTC NHPT dominant hand } & & 0.14 & & & \\
\hline & MBT_1 & & -0.81 & 0.23 & 0.001 \\
\hline \multirow[t]{2}{*}{ DTC NHPT non-dominant hand } & & 0.07 & & & \\
\hline & MBT_1 & & -0.49 & 0.20 & 0.02 \\
\hline \multirow[t]{2}{*}{ DTC MBT dominant hand } & & 0.08 & & & \\
\hline & MBT_3 & & 0.43 & 0.16 & 0.009 \\
\hline DTC MBT non-dominant hand & & $\begin{array}{l}\text { No variables entered } \\
\text { in the model }\end{array}$ & & & \\
\hline
\end{tabular}

DTC: dual-task cost; NHPT: nine-hole peg test; MBT: months backward test; MS: multiple sclerosis.

environment and by factors that minimize danger and maximize pleasure (Plummer and Eskes, 2015). For example, cutting vegetables may be interrupted when another person is asking something. Therefore, results may be different in a real-life setting.

The results of this study may support the bottleneck and the capacity-sharing model of the DTC (Pashler, 1994; Tombu and Jolicœur, 2003). When participants performed the dual task with their dominant hand, the physical task was prioritized. When performing the operation, the neural paths for executing the NHPT may inhibit the neural paths for executing the MBT. Another explanation may be the allocation of the mental fuel to the physical task instead of the mental task, resulting in prioritization of the physical task. This study did not explore neural correlates in dualtask conditions, although this may have provided more insight into these hypothesized models. The evidence for one model or the other to explain the DTC is limited and warrants future research (Leone et al., 2017).

The variability of the DTC of fine motor performance can partially be explained by the presence of MS. This finding could be related to disability status, although the evidence of the correlation between the DTC and disability status based on the EDSS is conflicting (Wajda and Sosnoff, 2015).
Among individuals with MS, cognitive performance is an important correlate of the DTC score of cognitive and fine motor performance, predominantly when the tasks are performed by the non-dominant hand. This is in line with the correlation of processing speed in cognitive-motor interference while walking (Motl et al., 2014).

For clinical practice, dual-task training may be considered, focusing on the cognitive aspect to MS individuals who have a low hand capacity. On the other hand, people with MS with high cognitive deficits, may benefit from dual-task training focusing on the physical aspect. The efficacy of dual-task training for postural control is promising, but interventions to improve the DTC of fine motor and cognitive performance in MS are lacking (Ghai et al., 2017; Wajda et al, 2017). Occupational therapists may provide people with MS and their caregivers with information about difficulties when dual tasking in daily activities, although the DTC of bimanual tasking and activities of daily life is still unclear and needs further investigation.

\section{Study limitations and strengths}

The results of this study may be influenced by selection bias. Controls were selected among the researchers' family 
members and neighbors. Because the participants knew the investigator, who was present for study sessions, performance may be influenced in a positive or negative way.

Participants were recruited by convenience sampling. This method of non-probability sampling may cause a less representative sample of the population. However, we used the database of both in and outpatients of the center representing a large proportion of rehabilitation users with MS in Belgium.

To determine hand dominance, participants had to mention their current writing hand. The use of a standardized measure such as the Edinburgh handedness inventory (Oldfield, 1971) would provide more objective information about hand dominance. Moreover, when testing the dual-task condition, the participants were asked to start with their dominant hand. Our results revealed that scores of the MBT in a dual-task condition were significantly better when simultaneously performed with the NHPT by the non-dominant hand. This result may be due to the fact that the participants always started the dual task with their dominant hand and experienced a learning effect on the MBT, despite the practice sessions. Randomization of the performance order of the dominant versus non-dominant hand in future studies may improve the interpretation of these results.

This study only used one test for the assessment of fine motor control and cognition, namely the NHPT and the MBT. Using multiple tests for fine motor and cognitive assessment would strengthen this study. In addition, including assessment of educational levels would improve the discussion of the results, as these may influence cognitive performance (Östberg et al., 2012).

Besides these limitations, our study has several strengths. The use of a considerable sample size is important to collect data from a heterogeneous population. In addition, this study explored different levels of MS by dividing the MS group into hand capacity and cognitive capacity groups, instead of focusing on the EDSS. This provides an additional dimension, which has not been studied before. Furthermore, this study partially closed the knowledge gap by adding evidence on the DTC of fine motor and cognitive performance over the DTC of walking and postural performance.

\section{Conclusion}

This study provides evidence for the DTC of fine motor and cognitive performance among people with MS and among hand capacity and cognitive capacity groups in MS. People with MS and healthy controls showed the DTC for the NHPT and MBT speed, not for MBT accuracy. In individuals with MS with a highly affected hand function, reduced cognitive performance in a dual-task condition was visible when executed with their most affected hand. Participants with MS with a highly affected cognition, showed a higher DTC for fine motor performance in both hands. Prioritizing was not different between MS and healthy controls. In general, participants prioritized the fine motor task, when this task was executed by the dominant hand. The total variability of the DTC of fine motor performance can partially be explained by the presence of MS. Among MS individuals, cognitive performance is an important correlate of the DTC of cognitive and fine motor performance when the dual task is performed by the non-dominant hand.

\section{Key findings}

- The DTC was not different between subjects with MS and controls. Individuals with MS with limited hand function showed an increased DTC of cognitive performance.

- Individuals with MS with lower cognitive capacity showed an increased DTC of fine motor performance.

\section{What the study has added}

The DTC of fine motor and cognitive performance among individuals with MS has not yet been widely explored. The results imply that occupational therapists should focus on the cognitive or the physical aspect during dual-task training dependent on individuals' capacities.

\section{Acknowledgements}

The authors wish to thank all participants for their time and effort in conducting the tests for this study.

\section{Research ethics}

This study was approved by the local ethical committee of the National MS Centre Melsbroek, Belgium (2016) and the ethical committee of the University Hospital Ghent, Belgium (2016, reference 2015/1503). All participants provided written informed consent.

\section{Declaration of conflicting interests}

The author(s) declared no potential conflicts of interest with respect to the research, authorship, and/or publication of this article.

\section{Funding}

The author(s) received no financial support for the research, authorship, and/or publication of this article.

\section{References}

Bertoni R, Lamers I, Chen CC, et al. (2015) Unilateral and bilateral upper limb dysfunction at body functions, activity and participation levels in people with multiple sclerosis. Multiple Sclerosis Journal 21(12): 1566-1574. https://doi.org/10.1177/ 1352458514567553.

Bjartmar C and Trapp BD (2001) Axonal and neuronal degeneration in multiple sclerosis: mechanisms and functional consequences. Current Opinion in Neurology 14(3): 271-278. https://doi.org/10.1097/00019052-200106000-00003.

Boringa JB, Lazeron RH, Reuling IE, et al. (2001) The brief repeatable battery of neuropsychological tests: normative values allow application in multiple sclerosis clinical practice. Multiple Sclerosis Journal 7(4): 263-267. https://doi.org/10. $1177 / 135245850100700409$.

Cunningham EL, Passmore AP, McAuley DF, et al. (2015) Reciting the months of the year backwards: what is a 
“normal” score? Age and Ageing 44(3): 537-538. https://doi. org/10.1093/ageing/afv056.

DeLuca GC, Yates RL, Beale H, et al. (2015) Cognitive impairment in multiple sclerosis: clinical, radiologic and pathologic insights. Brain Pathology 25(1): 79-98. https://doi.org/10. 1111/bpa.12220

Downer MB, Kirkland MC, Wallack EM, et al. (2016) Walking impairs cognitive performance among people with multiple sclerosis but not controls. Human Movement Science 49(2016): 124-131. https://doi.org/10.1016/j.humov.2016.06.010.

Faul F, Erdfelder E, Lang A-G, et al. (2007) G*Power 3: a flexible statistical power analysis program for the social, behavioral, and biomedical sciences. Behavior Research Methods 39(2): 175-191.

Ferdinand S, Vandenbroucke A, D'Hooge M, et al. (2015) Can we evaluate the influence of mental tasks on motor tasks in upper limb and vice versa in persons with MS? Multiple Sclerosis Journal 21(4): 496. https://doi.org/10.1177/135245 8515573128.

Ghai S, Ghai I and Effenberg A (2017) Effects of dual tasks and dual-task training on postural stability: a systematic review and meta-analysis. Clinical Interventions in Aging 12: $557-577$.

Hawkes CH and Giovannoni G (2010) The McDonald criteria for multiple sclerosis: time for clarification. Multiple Sclerosis 16(5): 566-575. https://doi.org/10.1177/1352458510362441.

Heremans E, D'Hooge A-M, De Bondt S, et al. (2012) The relation between cognitive and motor dysfunction and motor imagery ability in patients with multiple sclerosis. Multiple Sclerosis 18(9): 1303-1309. https://doi.org/10.1177/135245 8512437812 .

Lamers I, Kelchtermans S, Baert I, et al. (2014) Upper limb assessment in multiple sclerosis: a systematic review of outcome measures and their psychometric properties. Archives of Physical Medicine and Rehabilitation 95(6): 1184-1200. https://doi.org/10.1016/j.apmr.2014.02.023.

Lamers I, Kerkhofs L, Raats J, et al. (2013) Perceived and actual arm performance in multiple sclerosis: relationship with clinical tests according to hand dominance. Multiple Sclerosis 19(1): 1341-1348. https://doi.org/10.1177/1352458513475832.

LaRocca NG (2011) Impact of walking impairment in multiple sclerosis. The Patient: Patient-Centered Outcomes Research 4(3): 189-201. https://doi.org/10.2165/11591150-000000000-00000.

Learmonth Y, Ensari I and Motl R (2017) Cognitive motor interference in multiple sclerosis: insights from a systematic quantitative review. Archives of Physical Medicine and Rehabilitation 98(6): 1229-1240. https://doi.org/10.1016/j. apmr.2016.07.018

Learmonth YC, Pilutti LA and Motl RW (2015) Generalised cognitive motor interference in multiple sclerosis. Gait and Posture 42(1): 96-100. https://doi.org/10.1016/j.gaitpost. 2015.04.014.

Leone C, Feys P, Moumdjian L, et al. (2017) Cognitive-motor dual-task interference: a systematic review of neural correlates. Neuroscience and Biobehavioral Reviews 75(2017): 348-360. https://doi.org/10.1016/j.neubiorev.2017.01.010.

Leone C, Patti F and Feys P (2015) Measuring the cost of cognitive-motor dual tasking during walking in multiple sclerosis Multiple Sclerosis 21(2): 123-131. https://doi.org/10.1177/ 1352458514547408 .

Lindenberger U, Marsiske M and Baltes PB (2000) Memorizing while walking: increase in dual-task costs from young adulthood to old age. Psychology and Aging 15(3): 417-436. https:// doi.org/10.1037/0882-7974.15.3.417.
Malcolm BR, Foxe JJ, Butler JS, et al. (2015) The aging brain shows less flexible reallocation of cognitive resources during dual-task walking: a mobile brain/body imaging (MoBI) study. NeuroImage 117(2015): 230-242. https://doi.org/10. 1016/j.neuroimage.2015.05.028.

Mathiowetz V, Weber K, Kashman N, et al. (1985) Adult norms for the nine hole peg test of finger dexterity. Occupational Therapy Journal of Research 5(1): 24-38. https://doi.org/10. 1177/153944928500500102.

Motl RW, Sosnoff JJ, Dlugonski D, et al. (2014) Walking and cognition, but not symptoms, correlate with dual-task cost of walking in multiple sclerosis. Gait and Posture 39(3): 870-874. https://doi.org/10.1016/j.gaitpost.2013.11.023.

Navon D and Miller J (1987) Role of outcome conflict in dualtask interference. Journal of Experimental Psychology: Human Perception and Performance 13(3): 435-448. https://doi.org/ 10.1037/0096-1523.13.3.435.

Oldfield RC (1971) The assessment and analysis of handedness: the Edinburgh inventory. Neuropsychologia 9(1): 97-113. https://doi.org/10.1016/0028-3932(71)90067-4.

Östberg P, Hansson V, Häägg S, et al. (2012) Adult norms and test-retest reliability for the months backward test: durational and response accuracy measures. Logopedics, Phoniatrics, Vocology 37(1): 11-17. https://doi.org/10.3109/14015439. 2011.614957.

Pashler H (1994) Graded capacity-sharing in dual-task interference? Journal of Experimental Psychology: Human Perception and Performance 20(2): 330-342.

Plummer P and Eskes G (2015) Measuring treatment effects on dual-task performance: a framework for research and clinical practice. Frontiers in Human Neuroscience 9(2015). https://doi. org/10.3389/fnhum.2015.00225.

Polman C, Reingold S, Edan G, et al. (2005) Diagnostic criteria for multiple sclerosis: 2005 revisions to the "McDonald Criteria”. Annals of Neurology 58: 840-846.

Rao S (ed.) (1990) A Manual for the Brief, Repeatable Battery of Neuropsychological Tests in Multiple Sclerosis. New York: National Multiple Sclerosis Society.

Tomassini V, Johansen-Berg H, Leonardi L, et al. (2011) Preservation of motor skill learning in patients with multiple sclerosis. Multiple Sclerosis 17(1): 103-15. https://doi.org/10. $1177 / 1352458510381257$.

Tombu M and Jolicœur P (2003) A central capacity sharing model of dual-task performance. Journal of Experimental Psychology: Human Perception and Performance 29(1): 3-18. https://doi.org/10.1037/0096-1523.29.1.3.

von Elm E, Altman DG, Egger M, et al. (2008) The Strengthening the Reporting of Observational Studies in Epidemiology (STROBE) statement: guidelines for reporting observational studies. Journal of Clinical Epidemiology 61(4): 344-349. https://doi.org/10.1016/j.jclinepi.2007.11.008.

Wajda DA, Mirelman A, Hausdorff JM, et al. (2017) Intervention modalities for targeting cognitive-motor interference in individuals with neurodegenerative disease: a systematic review. Expert Review of Neurotherapeutics 17(3): 251-261. https://doi.org/10.1080/14737175.2016.1227704.

Wajda DA, Motl RW and Sosnoff JJ (2013) Dual-task cost of walking is related to fall risk in persons with multiple sclerosis. Journal of the Neurological Sciences 335(1-2): 160-163. https://doi.org/10.1016/j.jns.2013.09.021

Wajda DA and Sosnoff JJ (2015) Cognitive-motor interference in multiple sclerosis?: a systematic review of evidence, correlates, and consequences. BioMed Research International 2015(Article ID 720856): 8. https://doi.org/10.1155/2015/ 720856. 